\title{
The titanium PV I endosteal implant from beta-titanium alloy Ti 38Nb 6Ta
}

\author{
Patrik Prachar, Sonia Bartakova, Jiri Vanek
}

\begin{abstract}
Aim. The aim of this study was to use the beta-titanium alloy Ti38Nb6Ta for production of a new construction line of implants, perform testing on animals and preclinical tests.

Materials and Methods. Within this study, a new PV I implant with five construction variants was developed. The implant includes three types of threads - microthreads and flat threads of two types with a different depth. Further, the PV I implant was tested on minipigs. Subsequently, preclinical tests of 150 implants were performed and assessed. The age interval of patients was from 18 to 74 years.

Results. Beta titanium alloy exhibited higher strength than titanium alloys. Anti-corrosion resistance was also higher. The implant from beta-alloy was inserted in the tibias of minipigs. Sections showed good osseointegration of the PV I implant. During the preclinical tests, 150 implants were inserted with the success rate of $99.33 \%$ after the two year assessment. The assessment also included handicapped patients who are not usually assessed in classical studies. Finally, the implantation protocol and documentation of a new implantation system PV I was designed. At the same time the industrial sample of this implant was formed and accepted.

Conclusion. A new anti-rotation PV I implant with microthreads and conical anchorage of the abutment into the fixture was formed. The beta-titanium alloy Ti38Nb6Ta used for the implant was biocompatible and had higher mechanical and physical properties than the existing titanium alloys. The PV I implant was recommended for clinical application.
\end{abstract}

Key words: beta-titanium alloy, anti-rotation feature, microthreads, handicapped patient

Received: November 19, 2013; Accepted with revision: February 20, 2014; Available online: March 10, 2014 http://dx.doi.org/10.5507/bp.2014.011

Clinic of Stomatology, Faculty of Medicine, Masaryk University, Brno and St. Anne's University Hospital in Brno, Czech Republic Corresponding author: Patrik Prachar, e-mail: patrik.prachar@fnusa.cz

\section{INTRODUCTION}

Dental implantology has become a standard competence in treating dental defects. A number of dental implantation systems have been developed, namely endosteal using metal alloys and ceramics. Alloys of titanium Grade I-V with the addition of Al, V, Mo, Zr, Ta, Hf, Nb for the improvement of healing and functioning properties of these implants are preferred due to their biological and mechanical-physical properties ${ }^{1}$. Their specific characters lie in their construction, shape, anchorage of the abutment, surface modification and inserting instrumentarium ${ }^{2-4}$. The main objective of implantology is to achieve in a shortest possible time after insertion into the tissue the optimal permanent tissue integration in a wide range of clinical indications, also in handicapped and risky patients. A special attention has been paid to a shape of implants, shape modification of threads in relation to the bone, surface modification by coating or using some other treatment, e.g. electroerosion etc. ${ }^{3,4}$. In the two stage implants, perfect initial stability of the implant and perfect anchorage of the abutment are essential to avoid the implant loosening and prevent penetration of secondary infection. In relation to the above mentioned, it has been demonstrated that titanium alloys with the additional components of $\mathrm{Al}, \mathrm{V}$ are not biologically fully optimal $^{5}$ while the addition of $\mathrm{Nb}, \mathrm{Ta}, \mathrm{Zr}$, Mo, Hf have been confirmed as more suitable ${ }^{6}$. In addition, titanium alloy with a modified admixture of metal components must allow processing for achieving a required shape of the implant while keeping optimal mechanical and physical characters after the insertion into the soft tissues and bone (e.g. module of flexibility). Our previous studies have demonstrated suitability of the beta - microstructure of the basic titanium alloy ${ }^{5,7-9}$.

Within the activities of the SVC (Stomatological Research Center) in 2005 - 2011 aiming at achieving the above mentioned requirements, after continuous biophysical tests, beta-titanium alloy Ti38Nb6Ta was developed and filed for patent rights 6 . Subsequently, from this alloy five types of PV implants (PV I - PV V) with modified threads and perfect connection of the abutment into the fixture were newly designed. The tissue integration of these implants was checked experimentally in the scope of the international collaboration ${ }^{5}$. This study presents construction and first results from preclinical tests of PV I, which was the most type.

\section{MATERIAL AND METHODS}

The PV I implant was designed as a two-stage dental implant with anti-rotational and anti-peri-implantation features. It consists of a cylindrical fixture with a conical apical end (Fig. 1). The edge of the coronary part was beveled to allow a deeper insertion of the implant into the 


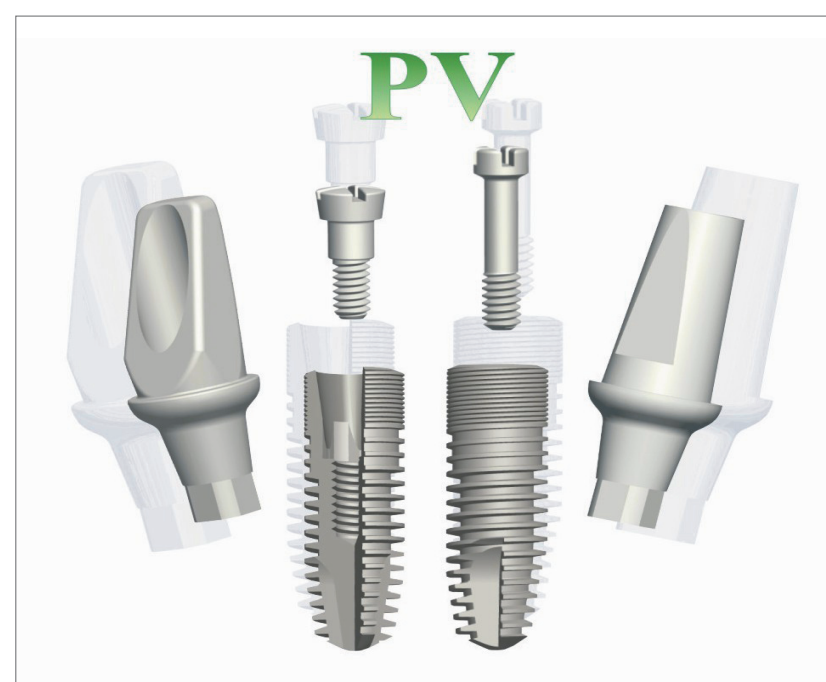

Fig. 1. Implantation system PV I.

bone and prevent a higher stress and associated resorption of the bone (corticalis). The threads in the fixture were pre-drilled and consisted of three parts:

The first - coronary part contained microthreads.

The second - threads of the middle part with a pitch of $0.6 \mathrm{~mm}$ and depth of $0.25 \mathrm{~mm}$

The third - apical part consisted of threads with a pitch of $0.6 \mathrm{~mm}$ and depth from 0.3 to $0.5 \mathrm{~mm}$.

The treads of the second and third parts of the implant had flat profiles, which classifies them as very good threads in terms of stress - bone ratio. Further, two trenches in the apical part allow condensation of the bone during the implant insertion. The coronary part of the implant was beveled to reduce the stress in the cortical bone and polished for a smooth contact with the soft tissue.

The inner part of the fixture contained a coil for screwing the abutment, square prism as an anti-rotation feature facilitating the insertion of the implant and stabilization of the abutment, and finally self-locking angel providing a firm implant-abutment connection and preventing the peri-implant disease development. The abutment consisted of a screw for fixation in the fixture, a square prism fitting into the fixture with conical broadening for a tight connection. The abutment itself in the gingival part allowed the least possible dead space to be formed in the transition area between the bone and gingiva.

Subsequently, testing on animals was conducted in collaboration between the Stomatological Research Centrum (SRC) and Tokyo Medical and Dental University. Oneyear old minipigs were used as laboratory animals as their bone tissue is similar to that of humans. The implants were introduced into the tibias of pigs and evaluated after a month on the sections. Testing of implants were at two minipigs. Now the variant with beta-alloy corresponded two implants. It was found that implant healing by osseointegration has been fully. This experiment was approved by the Ethical Commitee of Japan. This study assessed only the beta alloy implant sections.

Preclinical tests performed within the SRC project and other application tests included insertion of 150 implants

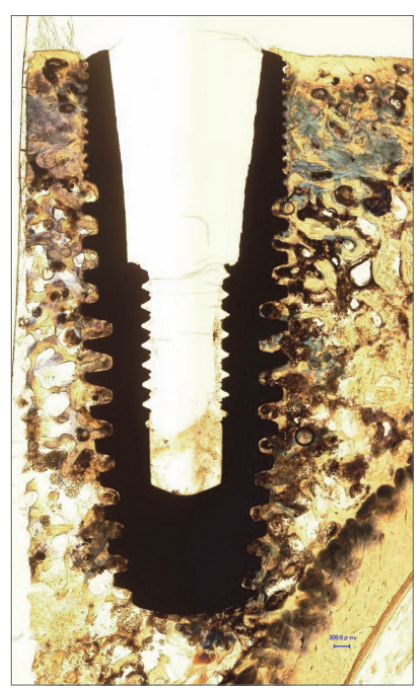

Fig. 2. Section of the tibia bone with the PV I. implant in a minipig.

of PV I type. Before implantation, each patient was informed about the preclinical testing and then enrolled into the protocol. The material for the pre-clinical tests was wholly bioinert.

The implants were used as solo pillars, in connection with teeth or without the tooth-implant connection and implants in the toothless jaw. Totally 83 implants in 37 females and 67 implants in 28 males were introduced. The age interval of patients was from 18 to 74 years.

\section{RESULTS}

The beta titanium alloy exhibited excellent construction and mechanical and physical properties. Compared to titanium alloys, higher strength was achieved ${ }^{10}$. In addition, anti-corrosion resistance was higher than in the titanium alloys ${ }^{10-12}$.

The insertion of the implant into the tibia of minipigs and evaluation of osseointegration were carried out in Japan. The implants were examined on the bone sections after one month (Fig. 2). In case of the implant from beta alloy, osseointegration did not exhibit any signs of resorption.

In the preclinical phase, the success rate of the placed implants was $99.33 \%$. This corresponds to one explanted implant removed still during the healing period. In this one case single stage augmentation was performed. There was not any antiinflammation reaction but a stable connection was not a result of osseointegration but fibrointegration. This could have been caused by an error during the insertion, i.e. insufficient primary stability of the implant. In females, the success rate of implantation was $100 \%$ in all indications; in males, the success rate of implantations in the indication of the "in-gapped" pillar in connection with the teeth was $95.00 \%$. The success rate of the other indications was $100 \%$. The high success rate reflects a short interval (the assessment after two years) (Table 1). Samples of some implantations are shown in RTG images (Fig. 3-5). 
Table 1. Number of implants according to the indications of insertion and the success rate of the implantation within two years.

\begin{tabular}{lrrrrcr}
\hline & Total / Success Rate & \multicolumn{2}{c}{ Females / Success Rate Males / Success Rate } \\
\hline Solo pillar & 22 & $100 \%$ & 15 & $100 \%$ & 7 & $100 \%$ \\
Shortened dental arch & 42 & $100 \%$ & 27 & $100 \%$ & 15 & $100 \%$ \\
"In-gapped" pillar in connection with teeth & 38 & $97.37 \%$ & 18 & $100 \%$ & 20 & $95 \%$ \\
Toothless jaw & 48 & $100 \%$ & 23 & $100 \%$ & 25 & $100 \%$ \\
Total no. of implants & 150 & $99.33 \%$ & 83 & $100 \%$ & 67 & $98.51 \%$ \\
\hline
\end{tabular}

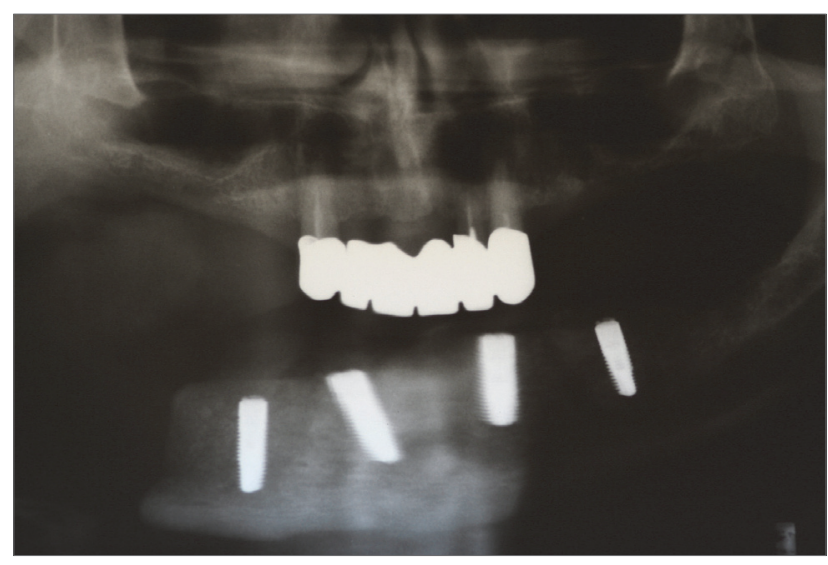

Fig. 3. State after the insertion of PV I implants in a patient after resection of the mandibula.

Finally, the implantation protocol and documentation of the new PV I implantation system were created (Fig. 6).

\section{DISCUSSION}

Biotests conducted in collaboration with Japan confirmed the optimal characters of the beta titanium alloy ${ }^{13}$ and excellent construction properties of the implants. The threads in the apical part were assessed as very good threads considering the stress-bone ratio ${ }^{14}$. Manipulation with the implants and their insertion was simple and accurate thus the PV I implants have a wide application not only in common indications but also in handicapped patients.

The construction in the cortical part - microthreads and beveled implant apix - ensured minimal resorption in the cortical part of the bone. This corresponds to similar threads used in the AstraTech system of the implants ${ }^{15-17}$.

The abutment, fully and accurately fitting the fixture is another positive feature of the implant construction. Its antirotation design ensures stability of the abutment and exact positioning by self-locking angel impedes penetration of bacteria ${ }^{18-20}$.

One failure occurred in this study shows the importance of the primary fixation of the implant in the bone in the augmentation of the sinus highmori for achieving implant stability. This case showed that the insufficient

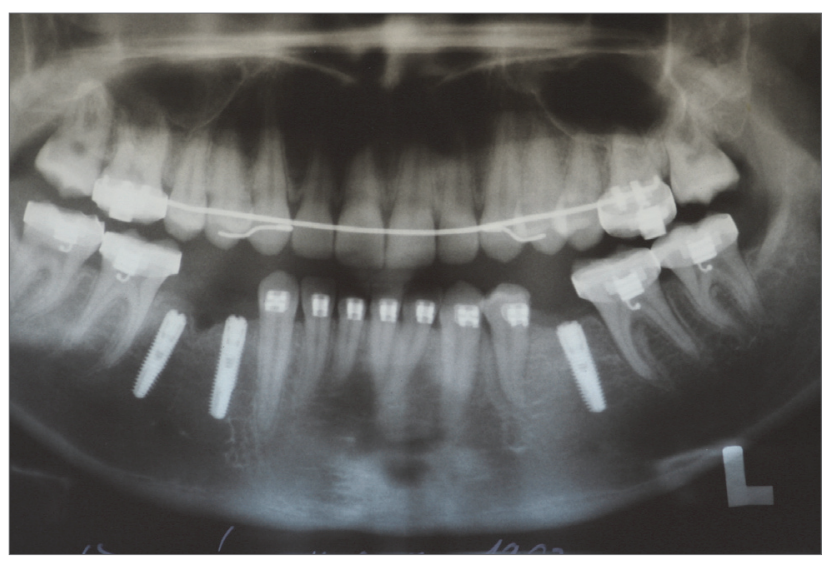

Fig. 4. State in a patient with hypodontia and a limit value of tolerance to titanium.

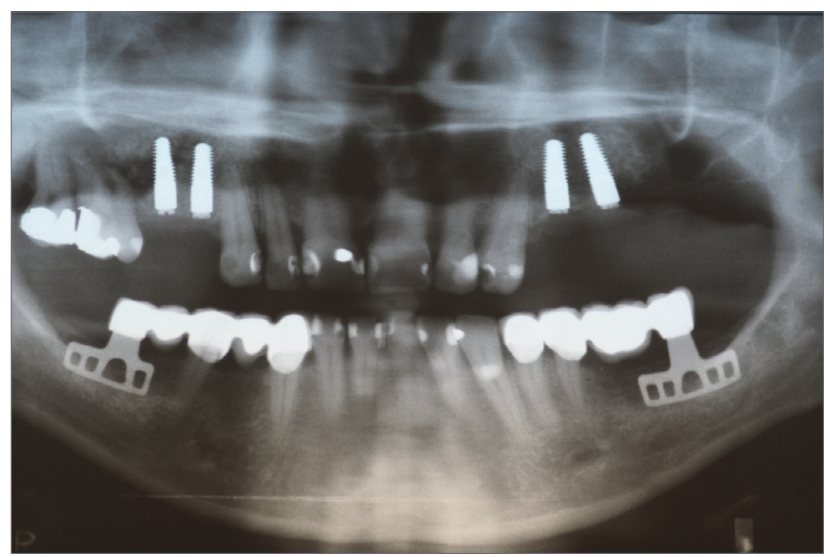

Fig. 5. State after the PV I implant insertion and bilateral augmentation of sinus highmori.

stability did not lead to required osseointegration thus the required result was not achieved.

Indications of the inserted implants also included risky subjects ( 24 implants in 7 patients), i.e. patients after resection of the mandibula, after radio- and chemotherapy and also patients with limit $(1.8-2.0)$ allergy to titan. In all these cases, the implantation and healing were without resorption and complications.

Therefore the PV I system but also other types of PV implants from beta alloy are perspective in terms of long term use in oral implantology. 


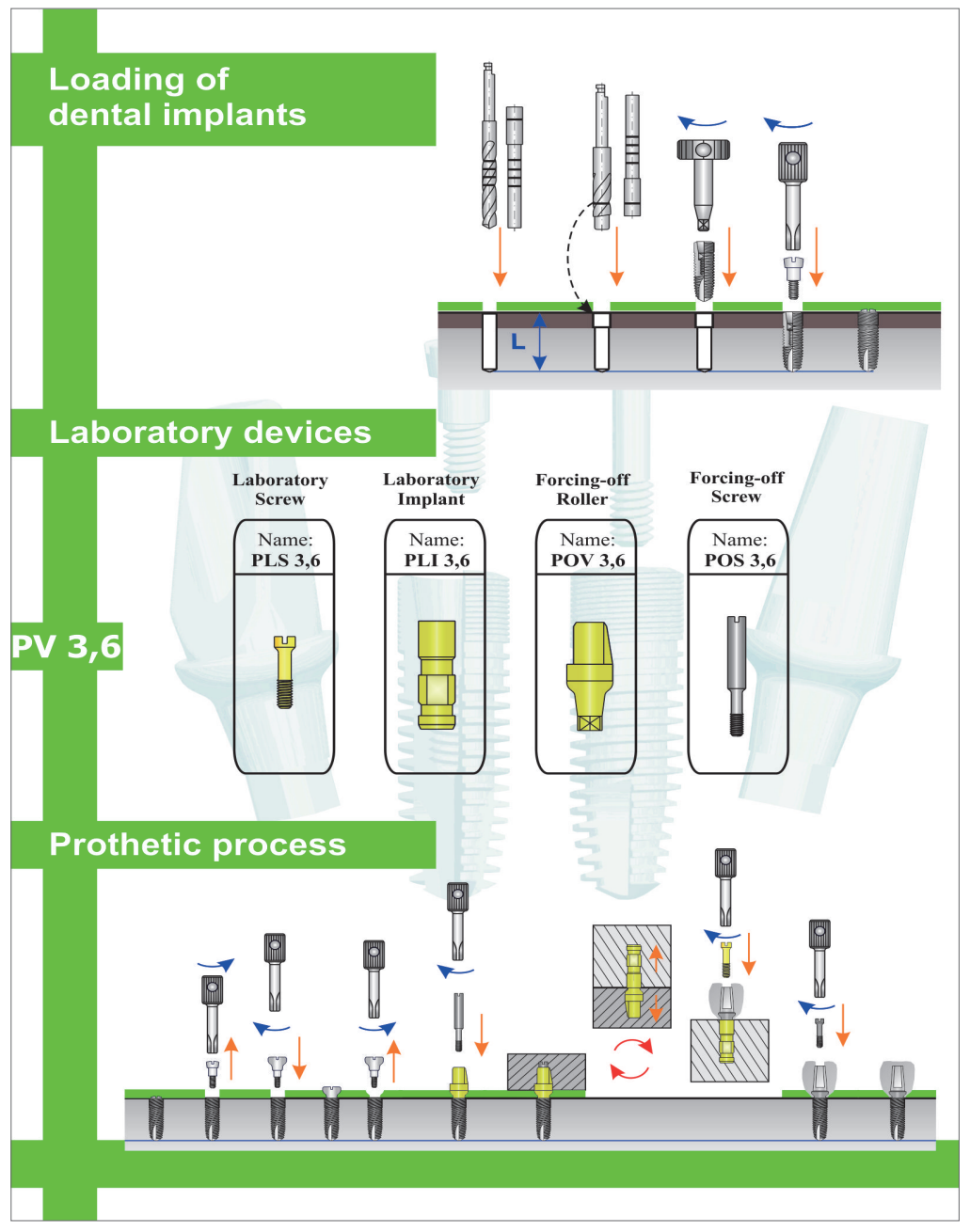

Fig. 6. Implantation protocol in the system PV I.

\section{CONCLUSION}

A new implant designated as PV I was developed. It is an anti-rotation implant of a partly conical shape with micro threads in the coronary part. It is anchored by locking angel for the minimal microbial invasion. Beta alloy used for implant manufacturing ensures biocompatibility and higher mechanical and physical properties compared to titanium alloy or pure titanium. Testing on minipigs did not prove any significant signs of resorption on the examined sections. Furthermore, pre-clinical assessment showed excellent results of the implant insertion. The results fully complied with our requirements for implants.

We can summarize that the implant system PV I made from beta alloy and its modification can be recommended for clinical application.

\section{ACKNOWLEDGEMENT}

This study was based on the project SVC 1M0528.

Author contributions: PP, SB, JV: literature search; PP, JV: manuscript writing; PP: study design; PP: data collection; PP, SB: data analzsis; PP: data interpretation; PP: statistical analysis; PP, SB: final approval.

Conflict of interest statement: The authors state that there are no conflicts of interest regarding the publication of this article.

\section{REFERENCES}

1. Geurtsen W. Biocompatibility of dental alloys. Crit Rev Oral Biol Med 2002;13(1):71-84

2. Hanawa T. Titanium and its oxide film: a substrate for formation of apatite, in The Bone-Biomaterial Interface, ed. J. E. Davies, University of Toronto Press, Toronto 1991 p. 49-61.

3. Prachár P, Vaněk J, Cvrček L, Bartáková S. Characterisctics of Surface Layer of Chromium Cobal Alloy Coated with Titatnium Nitride and Zirconium Nitride. Prakt Zub Lék 2005;53(4):65-7 (In Czech).

4. Silvennoinen R, Vetterl V, Hasoň S, Tuononen H, Silvennoinen M, Miller K, Cvrček L, Vaněk J, Prachár P. Sensing of human plasma fibrinogen on polished, chemically etched and carbon treated titanium surfaces by diffractive optical element based sensor. Optics Express 2008;16(14):10130-40.

5. Bartáková S, Prachár P, Strecha J, Podhorná B, Kudrman J, Hnilica F, Březina V, Vaněk J. Titanium alloys with iron admixtures: analysis of its material characteristics, biocompatibility and suitability for applications in dental implantology. Stomatolog 2009;2:19-24 (In Czech). 
6. Matsunoa H, Yokoyamaa A, Watarib F, Uob M, Kawasakia T. Biocompatibility and osteogenesis of refractory metal implants, titanium, hafnium, niobium, tantalum and rhenium. Biomaterials 2001;22(11):1253-62.

7. Bartáková S, Prachár P, Kudrman J, Podhorná B, Březina V, Strecha J. Binary titan-columbium alloys and their biocompatibility. Stomatolog 2009;1:39-42 (In Czech).

8. Bartáková S, Prachár P, Kudrman J, Podhorná B, Březina V. Binary titan-tantalum alloys and their biocompatibility. Prakt Zub Lék 2009;57(3):37-40 (In Czech).

9. Eisenbarth E, Velten D, Muller M, Thull R, Breme J. Biocompatibility of $\beta$-stabilizing elements of titanium alloys. Biomaterials 2004;25(26):5705-13.

10. Tsutsumi $Y$, Bartáková S, Prachár $P$, Yalatu S, Migita S, Igita $H$ Doi, Nomura N, Hanawa T. Long-Term Corrosion Behavior of Biocompatible Beta-Type Ti Alloy in Simulated Body Fuid. Journa of the electrochemical society 2012;159:435-440.

11. Joska L, Fojt J. Corrosion behaviour of titanium after short-term ex posure to an acidic environment containing fluoride ions. Journa of Materials Science: Materials in Medicine 2009;21(2):481-8.

12. Takada $\mathrm{Y}$, Nakajima H, Okuno O, Okabe T. Microstructure and corrosion behavior of bojary titanium alloys with beta-stabilizing elements. Dent Mater J 2001;20:34-52.
13. Filipi V, Březina V. Biological tests of beta titanium alloys for implantology. In International seminar SRC. Brno: Masarykova universita, 2008.76-7, ISBN 978-80-210-4596-5.

14. Dzan L, Henyš P, Čapek L, Šimůnek A. Makrodesign of Implant Types and Shapes of Threads Used and their Evaluation Using Finite Element Analysis. Čes Stomat 2013;113(4):88-94 (In Czech).

15. Hansson $S$, Werke $M$. The implant thread as a retention element in cortical bone: the effect of thread size and thread profile: a finite element study. J Biomechanics 2003;36:1247-58.

16. Lee DW, Choi YS, Park KH, Kim CS, Moon IS. Effect of microthread on the maintenance of marginal bone level: a 3-year prospective study. Clin Oral Impl Res 2007;18:465-70.

17. Norton M. An in vitro evaluation of the strength of an internal conical interface compared to a butt joint interface in implant design. Clin Oral Impl Res 1997;8:290-8.

18. Kocar M, Seme K, Hren NI. Characterization of the Normal Bacterial Flora in Peri-implant Sulci of Partially and Completely Edentulous Patients. Int J Oral Maxillofac Implants 2010;25(4):690-8.

19. Rabel A, Kohler SG. Microbiological study on the prognosis of immediate implant and periodontal disease. Mund Kiefer Gesichtschir 2006;10(1):7-13.

20. Romanos GE, Nentwig GH. Immediate functional loading in the maxilla using implants with platform switching: five-year results. Int J Oral Maxillofac Implants 2009;24(6):1106-12. 